INFLAMMATORY BOWEL DISEASE

\title{
Cost of illness of inflammatory bowel disease in the UK: a single centre retrospective study
}

\author{
A Bassi, S Dodd, P Williamson, K Bodger
}

Gut 2004;53:1471-1478. doi: 10.1136/gut.2004.041616

See end of article for authors' affiliations

.....................

Correspondence to: Dr K Bodger, University Department of Medicine, Clinical Sciences Centre, University Hospital

Aintree, Liverpool L9 7AL UK; kbodger@ liverpool.ac.uk

Revised version received 5 April 2004

Accepted for publication

8 April 2004
Background and aims: The potentially high costs of care associated with inflammatory bowel disease (IBD) are recognised but we have little knowledge of the scale, profile, or determinants of these costs in the UK. This study aimed to describe costs of illness for a group of IBD patients and determine factors associated with increased healthcare costs.

Setting: A university hospital serving a target population of approximately 330000.

Patients and methods: A six month cohort of IBD patients receiving any form of secondary care was identified, comprising 307 cases of ulcerative (or indeterminate) colitis and 172 cases of Crohn's disease. Demographic and clinical data were abstracted from clinical records and individual resource use was itemised for all attributable costs (including extraintestinal manifestations). Item costs were derived from national and local sources. Cost data were expressed as mean six month costs per patient (with 95\% confidence interval (Cl)) obtained using non-parametric bootstrapping. Determinants of cost were analysed using generalised linear regression modelling. A postal survey of patients was undertaken to examine indirect costs, out of pocket expenses, and primary care visits.

Results: Inpatient services (medical and/or surgical) were required by 67 patients (14\%) but accounted for $49 \%$ of total secondary care costs. Drug costs accounted for less than a quarter of total costs. Individual patient costs ranged from $£ 73$ to $£ 33254$ per six months. Mean $(95 \% \mathrm{Cl})$ six month costs per patient were $£ 1256(£ 988, £ 1721)$ for colitis and £1652 (£1221, £2239) for Crohn's disease. Hospitalisation, disease severity grade, and disease extent correlated positively with cost of illness but costs were independent of age or sex. Compared with quiescent cases of IBD, disease relapse was associated with a 2-3-fold increase in costs for non-hospitalised cases and a 20 -fold increase in costs for hospitalised cases. Survey data suggested average six month costs were $<£ 30$ per patient for primary care visits (both diseases) and median loss of earnings were $£ 239$ for colitis and $£ 299$ for Crohn's disease.

Conclusions: This study represents the first detailed characterisation of the scale and determinants of costs of illness for IBD in a British hospital. Hospitalisation affected a minority of sufferers but accounted for half of the total direct costs falling on the healthcare system.

\footnotetext{
C
} rohn's disease and ulcerative colitis, collectively known as inflammatory bowel diseases (IBD), are chronic conditions of unknown aetiology. The clinical spectrum of IBD is very wide and ranges from an asymptomatic quiescent state to life threatening severe illness. Although relatively uncommon and rarely fatal, these disorders typically affect people during their economically productive adult lives and may require extensive medical and surgical interventions over several decades of disease. ${ }^{12}$ The potential economic impact of managing IBD is therefore considerable. ${ }^{1-10}$

There is pressure within all healthcare systems to control expenditure and a growing acceptance of the need for more accurate information on the costs of different diseases. ${ }^{1}$ In the UK, detailed cost data for individual diseases are difficult to obtain owing to the absence of any systematic process for recording patient specific resource use. As a result, our knowledge of the level and profile of healthcare costs for IBD in Britain is remarkably limited. In other healthcare systems, the billing databases of third party health insurers have served as a source of information about resources consumed by individual patients with IBD. $^{6}$ However, the level of information contained in such databases is generally limited to basic demographics and broad diagnostic labels. More detailed studies from other countries have relied either on retrospective analyses of real patient data (describing resource use in a defined patient cohort), ${ }^{34-10}$ on theoretical mathematical modelling, ${ }^{7}$ or on extrapolation of various national surveys. ${ }^{4}$ However, there have been no detailed studies undertaken within the UK National Health Service.

Current therapies for IBD are often palliative rather than curative. Novel immunomodulatory agents are currently attracting much interest although their high acquisition cost has contributed to relatively restricted criteria for their approved use. Robust cost effectiveness data for rival IBD therapies is lacking as few therapeutic trials have incorporated the prospective collection of resource data. Furthermore, given the lack of published primary data for levels of resource use among IBD patients in the UK, the cost inputs used for theoretical modelling exercises have relied on subjective cost estimates rather than real patient data. This clearly raises questions about the validity of such analyses and their relevance to day to day practice.

The aims of the present study were: (i) to define the profile of costs incurred by a large group of patients with IBD attending a single UK centre and to correlate costs of care with demographics, disease type, and other clinical variables (disease severity and extent); (ii) to determine the financial cost of disease relapse, by comparing healthcare costs of patients with quiescent disease to that of patients suffering an acute exacerbation; and (iii) to estimate primary care

Abbreviations: IBD, inflammatory bowel disease; 5-ASA, 5aminosalicylic acid 
costs, indirect (productivity) losses, and out of pocket expenses for these patients by means of a postal survey.

\section{METHODS}

\section{Setting and perspective}

The setting for the present study was a 1200 bedded university hospital situated in the Northwest of England. The hospital serves an urban population of approximately 330000 local residents and spends approximately £118.5 million per year on delivering secondary care services. We used the prevalence approach to costing illness, quantifying the economic burden of IBD over a six month time frame (June 2000 to December 2000). Direct costs were estimated from the perspective of the UK National Health Service (a single payer provider organisation) and were calculated for all products and services consumed by a group of IBD patients receiving care at our institution.

\section{Patient cohort}

Any patient receiving secondary care for IBD during the study time frame was eligible for inclusion. Cases were identified retrospectively by performing manual and/or computer searches of various hospital databases for IBD specific terms (ulcerative colitis, Crohn's disease, colitis, proctitis, IBD). This involved searching and cross referencing a range of NHS data sources (outpatient clinic letters, hospital discharge coding systems, diagnostic reports from radiology, histopathology, and endoscopy departments). Initial database screening identified 620 patients with possible IBD. Hospital case records were available for review in 580 of these subjects $(94 \%)$.

A firm diagnosis of IBD (ulcerative colitis, Crohn's disease, or indeterminate colitis) was confirmed in 479 patients, based on previously published diagnostic criteria. ${ }^{3}$ Patients with a range of other diagnoses (for example, infectious colitis or diverticulitis) were excluded. The final study population comprised a total of 479 cases of confirmed IBD who attended the hospital for some form of care at least once during the time frame of the study. This included 433 prevalent cases (ulcerative colitis, $\mathrm{n}=253$; Crohn's disease, $\mathrm{n}=160$; indeterminate colitis, $\mathrm{n}=20$ ) in whom the diagnosis had been established prior to the study start date, and 46 incident (new) cases (ulcerative colitis, $\mathrm{n}=31$; Crohn's disease, $n=12$; indeterminate colitis, $n=3$ ) in whom the diagnosis was made for the first time at some point during the study period.

\section{Clinical and demographic data}

Details of demographic and diagnostic information were abstracted from the case records. This included patient age, sex, type of IBD (ulcerative colitis, Crohn's disease, or indeterminate colitis), disease extent, and severity. For ulcerative colitis, disease extent was coded as either proctitis (distal to the rectosigmoid junction), left sided colitis (distal to the hepatic flexure), pancolitis, or "unknown" (if staging investigations were incomplete or equivocal). For Crohn's disease, disease stage was designated as ileal, colonic, ileocolonic, other, or "unknown". Cases of indeterminate colitis were coded as either left sided (anywhere distal to the hepatic flexure) or pancolitis.

A simplified disease severity scale was employed, as previously applied to retrospective data for Crohn's disease patients. ${ }^{3}$ This scale defines disease severity by the intensity of medical treatment, using seven discrete states ( 1 , remission; 2, mild disease; 3, severe disease, drug responsive; 4, severe disease, drug dependant; 5, severe disease, drug refractory; 6, surgery; and 7, post surgical remission). For each patient, minimum and maximum disease severity over the study time frame was estimated.

\section{Secondary care resource use}

Information relating to resource use over the course of the study period was abstracted from the clinical records. Only IBD related resource use was included ("attributable" costs) which included care for extraintestinal manifestations (for example, rheumatological services) but excluded episodes of treatment for unrelated comorbidity. Products and services were listed under the following categories.

- Outpatient services: number and type of ambulatory visits, including consultations with medical/surgical staff, dieticians, and stoma nurses.

- Inpatient services: ward type (medical, surgical, or intensive care) and duration of stay.

- Investigations: number and type of blood tests, microbiological tests, radiological procedures, and endoscopic procedures (including number of biopsy samples processed for histopathology).

- Medication: new prescriptions and long term (maintenance) treatments for IBD were noted and the total number of daily doses taken over the six month time frame was estimated. This included all IBD specific drugs (for example, aminosalicylic acid derivatives, corticosteroids, topical (rectal) therapies, and other immunomodulators), antibiotics, symptomatic remedies (for example, antidiarrhoeals and antispasmodics), analgesics, and treatments for specific extraintestinal features (for example, osteoporosis). Reducing doses of steroids were calculated on the basis of an average standard regimen unless deviation from the routine was clearly documented in the case notes. No allowance for patient compliance was made, such that maintenance therapies were assumed to continue without interruption over the study period. For inpatients, drug use was taken directly from the prescription chart.

- Surgery: details of the type of operation and the recorded duration (hours) of each operation was obtained.

\section{Direct cost estimates for secondary care products and services}

For each patient, direct expenditure (per six months) for individual products and services was derived by multiplying units of resource use by their unit cost. The sources used for deriving unit costs were the NHS national reference costs, Personal Social Services Research Unit, ${ }^{11}$ and costs derived from local sources. Values are expressed in 2000-2001 UK pounds sterling $(£)$. The cost estimates include staff salaries and training, heating and lighting, pharmacy services, and miscellaneous costs (for example, patient appliances, staff uniforms, patients clothing, hardware and crockery, bedding and linen, printing and stationary, cleaning, and office equipment, etc). Expenditure returns also incorporate an overheads element to reflect the cost of capital and support services in the provision of hospital services.

Item costs for laboratory tests (haematology, biochemistry, and microbiology) were based on departmental finance estimates that include the cost of technicians, consumables, supervising physician, and overheads. Medication costs were based on Monthly Index of Medical Specialities. Surgical procedure costs were derived from the product of operation time and staff hourly rates ${ }^{11}$ plus consumables and overheads. Patient lost work time was valued using national average wages. ${ }^{12}$

\section{Costs of routine care versus active disease management}

It is recognised that a significant number of IBD patients attending secondary care clinics have clinically quiescent 
Table 1 Demographic and clinical characteristics of a six month cohort of inflammatory bowel disease patients $(n=479)$ receiving care at a single centre

\begin{tabular}{|c|c|c|c|}
\hline Parameter & Ulcerative colitis & Crohn's disease & $\begin{array}{l}\text { Indeterminate } \\
\text { colitis }\end{array}$ \\
\hline \multicolumn{4}{|l|}{ No of patients } \\
\hline Prevalent cases & 253 & 160 & 20 \\
\hline Incident cases & 31 & 12 & 3 \\
\hline Age (y) (mean (SD)) & $52(17)$ & $46(17)$ & $47(18)$ \\
\hline Sex (male $n(\%))$ & $159(56)$ & $71(41)$ & $12(52)$ \\
\hline \multirow[t]{4}{*}{ Extent of disease (n (\%)) } & Proctitis: 55 (19) & lleal: $67(39)$ & Left sided: 18 (78) \\
\hline & Left sided: 133 (47) & Colonic: 54 (31) & Pancolitis: 2 (9) \\
\hline & Pancolitis: 79 (28) & lleocolonic: 48 (28) & Unknown: 3 (13) \\
\hline & Unknown: $17(6)$ & $\begin{array}{l}\text { Other: None } \\
\text { Unknown } 3(2)\end{array}$ & \\
\hline \multicolumn{4}{|c|}{ Extraintestinal complications (n (\%)) } \\
\hline Sclerosing cholangitis & $4(1.4)$ & $1(0.6)$ & \\
\hline Iritis/uveitis & $3(1)$ & $1(0.6)$ & \\
\hline Sacroileitis/AS/arthritis & $3(1)$ & $8(4.6)$ & \\
\hline Pyoderma/EN & 3 (1) & $1(0.6)$ & \\
\hline \multicolumn{4}{|l|}{ Max disease severity (n (\%)) } \\
\hline Remission & $27(9)$ & $12(7)$ & $6(26)$ \\
\hline Mild & $163(57)$ & $57(33)$ & $14(61)$ \\
\hline Severe* & $80(28)$ & $73(42)$ & $3(13)$ \\
\hline Surgery & 7 (3) & $13(8)$ & \\
\hline Post-Sx remission & 7 (3) & $17(10)$ & \\
\hline Previous surgery (n (\%)) & $7(3)$ & $44(28)$ & \\
\hline \multicolumn{4}{|l|}{ Hospitalised (n (\%)) } \\
\hline Without surgery & $28(10)$ & $18(10)$ & $1(4)$ \\
\hline With surgery & $7(2.5)$ & $13(7.5)$ & \\
\hline
\end{tabular}

*Severe category includes drug responsive, drug dependant, and drug refractory disease.

AS, ankylosing spondylitis; EN, erythema nodosum; Post-Sx remission, post surgical remission.

disease and may require no significant alteration in management during routinely scheduled follow up visits. The cost effectiveness of routine follow up has been questioned. ${ }^{13}$ We wished to quantify the costs associated with routine care and determine the incremental costs associated with managing more active disease. Patients with quiescent disease were defined as those who fulfilled the following criteria during the course of the study period: (a) no change in disease severity grade; (b) no requirement for immunosuppressants (corticosteroids or azathioprine); (c) no change in drug therapy; and (d) no specialist investigations (except routine blood tests and surveillance colonoscopy).

\begin{tabular}{|c|c|c|c|c|}
\hline \multirow[b]{2}{*}{ Parameter (per 6 months) } & \multicolumn{2}{|c|}{ Ulcerative colitis* } & \multicolumn{2}{|c|}{ Crohn's disease } \\
\hline & $\begin{array}{l}\text { Ambulatory } \\
\text { group } \\
(\mathrm{n}=241) \dagger\end{array}$ & $\begin{array}{l}\text { Hospitalised } \\
\text { group } \\
(n=27) \dagger\end{array}$ & $\begin{array}{l}\text { Ambulatory } \\
\text { group } \\
(n=130) t\end{array}$ & $\begin{array}{l}\text { Hospitalised } \\
\text { group } \\
(\mathrm{n}=28) \dagger\end{array}$ \\
\hline \multicolumn{5}{|l|}{ Outpatient services (visits) } \\
\hline IBD related & $1.8(0-8)$ & $2.8(0-3)$ & $2.2(0-7)$ & $2.9 ;(0-8)$ \\
\hline Extraintestinal & $1.14(1-2)$ & - & $1.25(1-3)$ & \\
\hline Dietician & $0.01(0-1)$ & - & $0.07(0-3)$ & $0.1(0-1)$ \\
\hline Stoma nurse & $0.01(0-1)$ & $0.07(0-1)$ & - & $0.03(0-1)$ \\
\hline Laboratory & $5.9(0-55)$ & $45.1(5-130)$ & $7.6(0-28)$ & $35.3(9-66)$ \\
\hline \multicolumn{5}{|l|}{ Radiology } \\
\hline Plain $x$ ray & $0.01(0-1)$ & $2.1(0-6)$ & $0.07(0-1)$ & $1.4(0-4)$ \\
\hline Barium enema & $0.02(0-1)$ & $0.03(0-1)$ & $0.01(0-1)$ & $0.07(0-1)$ \\
\hline Barium F/T & $0.01(0-1)$ & $0.07(0-1)$ & $0.1(0-1)$ & $0.30(0-2)$ \\
\hline USS Abd & $0.02(0-1)$ & $0.07(0-1)$ & $0.02(0-1)$ & $0.18(0-1)$ \\
\hline CT abdomen/pelvis & - & $0.07(0-1)$ & $0.01(0-1)$ & $0.01(0-1)$ \\
\hline MRI abdomen/Pelvis & - & - & - & $0.07(0-1)$ \\
\hline WBC scan & - & - & $0.01(0-1)$ & $0.07(0-1)$ \\
\hline DEXA scan & $0.03(0-1)$ & - & $0.07(0-1)$ & - \\
\hline Fistulogram & & & $0.01(0-1)$ & \\
\hline \multicolumn{5}{|l|}{ Endoscopies } \\
\hline OGD & $0.02(0-1)$ & $0.04(0-1)$ & $0.15(0-1)$ & $0.11(0-1)$ \\
\hline Sigmoidoscopy & $0.14(0-2)$ & $0.7(0-4)$ & $0.05(0-2)$ & $0.18(0-1)$ \\
\hline Colonoscopy & $0.18(0-1)$ & $0.3(0-3)$ & $0.1(0-1)$ & $0.3(0-3)$ \\
\hline \multicolumn{5}{|l|}{ Hospital admission } \\
\hline $\begin{array}{l}\text { No of admissions } \\
\text { Length of each admission (days) }\end{array}$ & NA & $\begin{array}{l}1.3(1-3) \\
18(1-32)\end{array}$ & NA & $\begin{array}{l}1.1(1-2) \\
14(4-40)\end{array}$ \\
\hline \multicolumn{5}{|c|}{$\begin{array}{l}\text { Values are mean (range). } \\
\text { Barium } \mathrm{F} / \mathrm{T} \text {, barium follow through; USS Abd, ultrasound of the abdomen; } \mathrm{CT} \text {, computed tomography; MRI } \\
\text { magnetic resonance imaging; WBC, white blood cell; OGD, oesophagogastroduodenoscopy. } \\
\text { *Includes patients with indeterminate colitis. } \\
\text { tExcludes incidence cases and those patients who were lost to follow up. }\end{array}$} \\
\hline
\end{tabular}




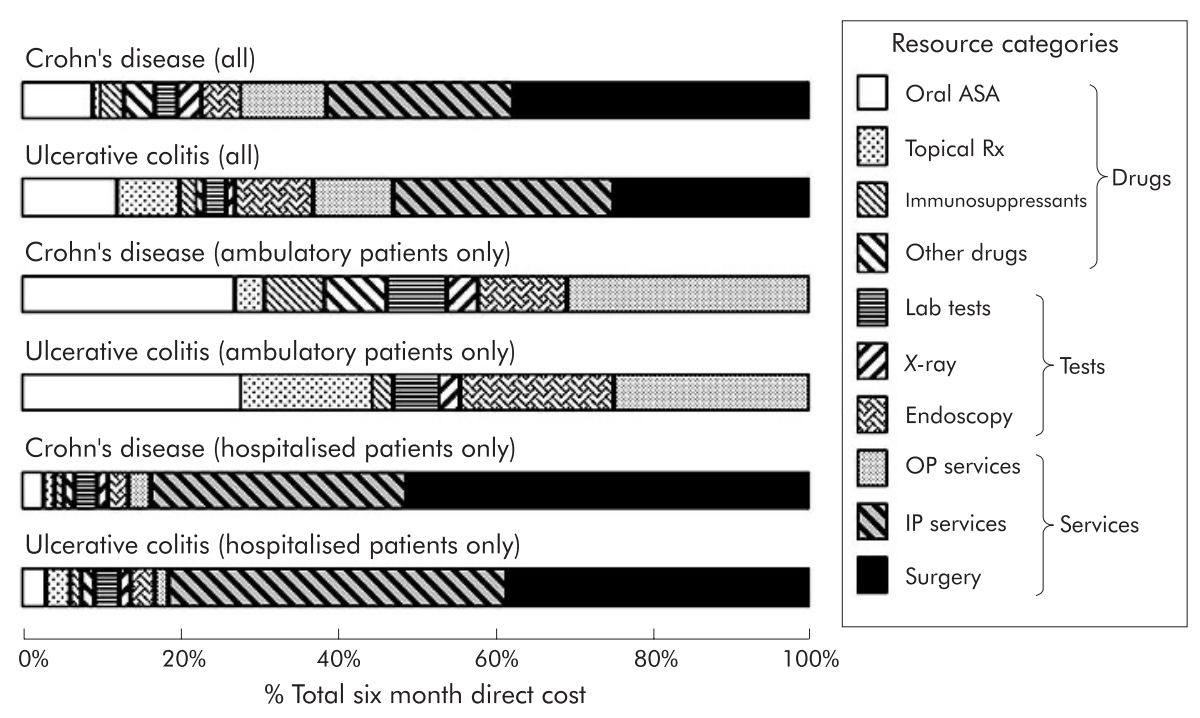

Figure 1 Contribution of different items and services to six month direct costs of care for inflammatory bowel disease patients (as per cent of totals). ASA, aminosalicylic acids; OP, outpatient; IP, inpatient.

These criteria were met by 118 patients with ulcerative (or indeterminate) colitis and 55 patients with Crohn's disease. Six month costs for subjects with quiescent disease were compared with those of patients experiencing a disease flare up. We defined a disease flare up as a transition from mild disease (no treatment, 5-ASA maintenance treatment alone, or topical treatment only) to a more severe disease state that required either outpatient immunosuppressant therapy or hospitalisation. Using this definition, a disease flare was experienced by 43 patients with ulcerative (or indeterminate) colitis and 30 patients with Crohn's disease.

\section{Postal survey}

A postal questionnaire was sent out to all confirmed IBD cases, requesting information relating to the previous six months for the following items: (1) number of IBD related visits to a primary care doctor (general practitioner); (2) estimated total IBD related out of pocket expenses (for example, for travel, prescription charges, special diets, or clothing); (3) employment status, and number of lost working days due to IBD; and (4) loss of "social" days (household and recreational activities).

\section{Data analysis and statistical methods}

Costs are given to the nearest pound sterling. Despite the skewed nature of cost data, it is desirable to report mean patient costs, as overall total costs can then be calculated. For this reason, mean patient costs per six months are reported with 95\% confidence intervals (CI) estimated using nonparametric bootstrap sampling. ${ }^{14}$ Two thousand samples were taken for each confidence interval and bias corrected and accelerated confidence intervals are presented, which correct for the difference between the observed means and the median of their bootstrap distribution and for the positive correlation observed between the bootstrap estimates and their estimated standard errors. Mann-Whitney U tests or the $\chi^{2}$ test for trend was used to compare rates of ambulatory visits, radiology services, and endoscopies between the diagnosis groups. Generalised linear regression, with a gamma error distribution and log link, was used to model direct costs, using a forward stepwise selection procedure (criteria for entry $\mathrm{p}<0.05$, and for removal $\mathrm{p}>0.1$ ) to select significant predictors of costs. ${ }^{15}$ The assumptions of the model were tested by examination of residuals and the modified Hosmer-Lemeshow test, ${ }^{16}$ and the appropriateness of the link and error distribution tested using the Box-Cox ${ }^{17}$ and Park $^{18}$ tests, respectively. Analysis was carried out using Stata version 8.0.

\section{RESULTS}

\section{Patient characteristics}

Demographic and clinical features of 479 IBD patients are summarised in table 1 . Based on an approximate referral population of 330000 people and the assumption that all eligible cases are referred to the centre, crude annual incidence and prevalence rates for ulcerative colitis were 19 per $10^{5}$ and 153 per $10^{5}$, respectively. Corresponding estimates for Crohn's disease were 8 per $10^{5}$ and 97 per $10^{5}$, respectively.

Sixty seven patients (14\%) were hospitalised during the study period (table 1). Extraintestinal manifestations were documented in $4.5 \%$ of cases of ulcerative colitis and $6.4 \%$ of Crohn's disease patients (table 1). Colon cancer had previously affected $0.8 \%$ of ulcerative colitis patients. One new tumour was diagnosed during the study time frame ( 1 cancer per 176.75 patient years of follow up). Two patients died during the study period (both with severe colitis associated with major comorbid illness) equivalent to a mortality rate of $0.8 \%$ per annum for the cohort. Two patients moved out of the region during the study period and further information on resource use was therefore unavailable.

\section{Secondary care resource use}

The various products and services utilised by the six month cohort of IBD patients are shown in table 2. Table 2 relates to prevalent cases only and excludes incidence cases (having less than six months of follow up) and patients who were lost to follow up. Consultation rates (ambulatory visits) were higher for Crohn's disease than for ulcerative colitis $(\mathrm{Z}=-2.992, \mathrm{p}=0.003)$. Endoscopy rate was slightly higher for ulcerative colitis than for Crohn's disease $\left(\chi^{2}(1)=3.7\right.$, $\mathrm{p}=0.055$ ) whereas the opposite was true for the use of radiology services $\left(\chi^{2}(1)=26.8, p<0.0001\right)$. Consultations with other specialities for the management of extraintestinal disease manifestations accounted for $2.4 \%$ of total ambulatory visits. Duration of stay during any one admission for hospitalised patients varied between 1 and 40 days. There were a total of 448 bed days on medical wards for ulcerative 


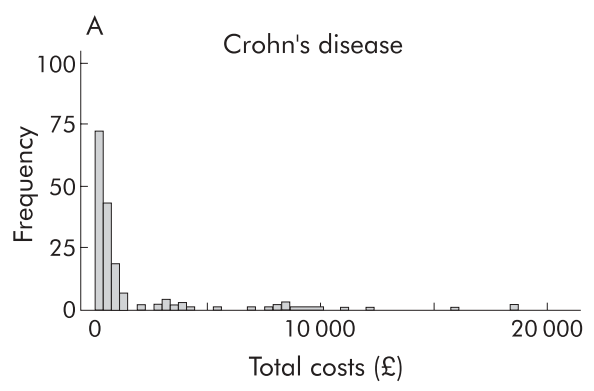

colitis and 260 bed days for Crohn's disease. Corresponding values for surgical bed days were 221 and 196, respectively. Surgical procedures included panproctocolectomy with ileostomy or anal pouch formation, extended right hemicolectomy, drainage procedures (for example, perineum), sigmoid colectomy, and ileal resection. Duration of surgery ranged from 0.5 to 6 hours. There were 12 days of intensive care bed occupancy.

\section{Secondary care costs}

The total cost of care for the entire IBD cohort over the study period was $£ 757433$ (Crohn's disease $£ 293$ 773; ulcerative or indeterminate colitis $£ 463660$ ). Total cost for patients receiving only ambulatory care was £240 615 (Crohn's disease $£ 75$ 195; ulcerative or indeterminate colitis $£ 165421)$ whereas the six month cost for patients in the hospitalised group was $£ 516817$ (Crohn's disease $£ 218$ 578; ulcerative or indeterminate colitis $£ 298$ 239).

The relative contribution of each cost item or service category to total expenditure is summarised in fig 1. Data are shown for all patients and separately for ambulatory and hospitalised groups. Interestingly, the distribution of costs between different items or services for the two forms of IBD was very similar. Overall, hospitalisation costs (inpatient services plus surgery) accounted for approximately half the total costs for each form of IBD. Drug costs accounted for less than a quarter of total costs. Oral 5-ASA preparations were the most significant contributor to drug costs, accounting for approximately half of the drugs bill. Although only three patients with Crohn's disease (1.7\%) received primary enteral nutritional therapy during the course of the study, their elemental feeds accounted for $10 \%$ of the total drugs bill. None of the patients with Crohn's disease received antitumour necrosis factor $\alpha$ therapy during the study time frame.

Radiological procedures accounted for a greater percentage of total costs for Crohn's disease $(2.5 \%)$ than for ulcerative (or indeterminate) colitis $(1.07 \%)$ whereas the opposite was

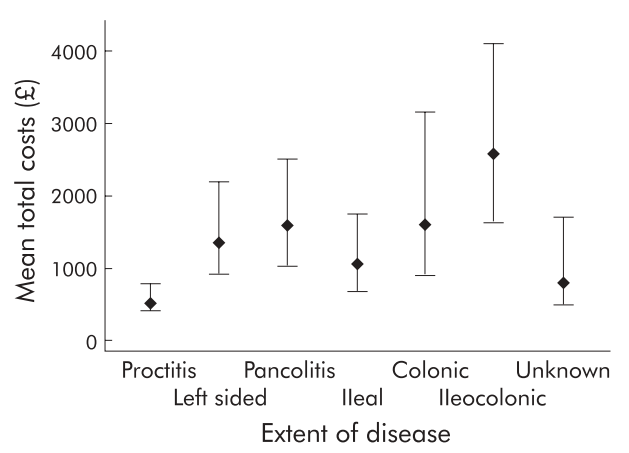

Figure 4 Mean (95\% confidence interval) six month costs versus disease extent.

observed with endoscopic procedures (4.6\% $\quad v \quad 9.7 \%)$. Surveillance colonoscopy accounted for $4 \%$ of endoscopy costs $(0.4 \%$ of total costs of care for IBD).

Figure 2 provides a frequency distribution for individual patient costs and shows a highly skewed profile. The top (most costly) $10 \%$ of patients accounted for $62 \%$ of total costs for ulcerative colitis and 59\% of total costs for Crohn's disease. Six month direct costs ranged from £73 to £33 254 for an individual patient.

\section{Mean costs and variation with disease type, severity, and extent}

Mean costs and (non-parametric bootstrap bias corrected and accelerated) $95 \%$ confidence intervals were calculated for patients who had a complete six month period of follow up (that is, "prevalent" cases only). Mean (95\% CI) six month cost per patient was $£ 1652$ (£1221, £2239) for Crohn's disease and $£ 1256$ ( $£ 988, £ 1721$ ) for ulcerative (or indeterminate) colitis. The corresponding six month costs for the ambulatory group alone was $£ 516$ ( $£ 452, £ 618)$ and $£ 539$ ( $£ 497, £ 589$ ), respectively. For the hospitalised group, mean six month

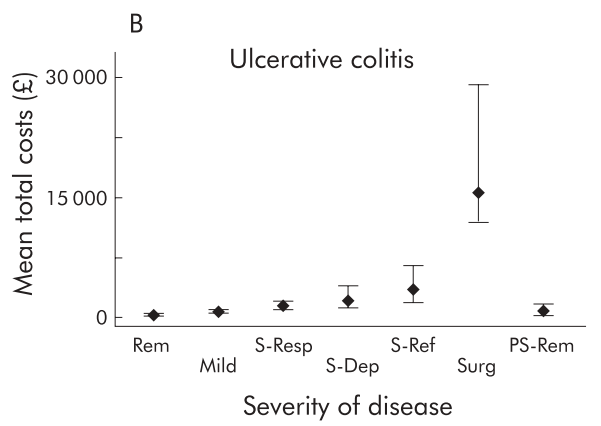

Figure 3 Mean (95\% confidence interval) six month costs versus disease severity in Crohn's disease (A) and ulcerative colitis (B). Rem, remission; Mild, mild disease; S-Resp, severe disease, drug responsive; S-Dep, severe disease, drug dependent; S-Ref, severe disease, drug refractory; Surg, surgery; PS, rem; post surgical remission. 
Table 3 Multivariate generalised linear modelling: influence of disease severity, hospitalisation, and diagnosis on total six month resource use for a cohort of inflammatory bowel disease patients

\begin{tabular}{|c|c|c|c|}
\hline Variable & Coefficient (SE) & $\begin{array}{l}\text { Relative increase in } \\
\text { costs associated with } \\
\text { variable (baseline }=1 \text { ) }\end{array}$ & p Value \\
\hline Diagnosis $†$ & $0.17(0.07)$ & $1.18(1.03,1.35)$ & 0.014 \\
\hline Hospitalisation & $2.04(0.12)$ & $7.65(6.11,9.60)$ & $<0.001$ \\
\hline Disease severity $\ddagger$ & & & $<0.001$ \\
\hline Mild disease & $1.03(0.11)$ & $2.80(2.26,3.47)$ & \\
\hline Severe disease, drug responsive & $1.25(0.14)$ & $3.47(2.66,4.54)$ & \\
\hline Severe disease, drug dependent & $1.64(0.13)$ & $5.13(3.99,6.60)$ & \\
\hline Severe disease, drug refractory & $1.63(0.16)$ & $5.09(3.71,7.00)$ & \\
\hline Surgery & $2.23(0.21)$ & $9.32(6.20,13.99)$ & \\
\hline Post surgical remission & $0.13(0.16)$ & $1.14(0.83,1.57)$ & \\
\hline Constant & $5.05(0.11)$ & & $<0.001$ \\
\hline
\end{tabular}

costs were $£ 6923$ (£5415, £8919) for Crohn's disease and $£ 7658$ (£5693, £10 651) for ulcerative (or indeterminate) colitis.

As expected, for both diseases there was an incremental increase in costs with worsening disease severity grade (fig 3 ). Mean (95\% CI) costs according to disease extent is shown in fig 4. Generalised linear modelling with a gamma distribution and log link was used to assess the importance of age, sex, diagnosis (Crohn's disease or ulcerative (including indeterminate) colitis), hospitalisation, and disease extent and severity in predicting overall costs of those with complete six month follow up (table 3). Age, sex, and disease extent were not found to be significant predictors of total costs once hospitalisation, diagnosis, and disease severity were accounted for.

Whereas the calculated "average" costs of care for Crohn's disease were higher than for ulcerative colitis, this reflects the higher rate of hospitalisation and surgery in the Crohn's disease group (table 1). The multivariate model predicted that ulcerative (or indeterminate) colitis patients had costs that were on average $18 \%(95 \%$ CI 3, 35\%) higher than patients with Crohn's disease. This reflects the fact that the model evaluates the independent influence of disease type on costs of illness by removing the confounding influence of disease severity and hospitalisation. In other words, the model adjusts for unequal case mix.

Total six month costs were estimated to be on average 7.65 (95\% CI 6.11, 9.60) times greater for hospitalised compared with ambulatory patients. The proportion of variation in the data explained by this model is approximately $77 \%$ (Efron's pseudo- $r^{219}$ ) and inspection of residuals and the scaled

Table 4 Postal survey: characteristics of responders and non-responders

\begin{tabular}{lcc}
\hline Parameter & $\begin{array}{l}\text { Responders } \\
(\mathbf{n}=233)\end{array}$ & $\begin{array}{l}\text { Non-responders } \\
(\mathbf{n}=246)\end{array}$ \\
\hline $\begin{array}{l}\text { Age (y) (mean (SD) } \\
\text { Sex (male, n (\%)) }\end{array}$ & $51(17)$ & $48(18)$ \\
Extent of disease (n (\%)) & $110(47)$ & $132(53)$ \\
$\quad$ Proctitis 27 (19) & $27(11)$ & $28(11)$ \\
Left sided 58 (47) & $58(25)$ & $75(30)$ \\
Pancolitis 37 (28) & $37(16)$ & $42(17)$ \\
lleal 67 (39) & $34(15)$ & $33(13)$ \\
Colonic 54 (31) & $25(11)$ & $29(12)$ \\
lleocolonic 48 (28) & $28(12)$ & $20(8)$ \\
Indeterminate colitis & $12(5)$ & $8(4)$ \\
Extent unknown & $12(5)$ & $11(4)$ \\
\hline
\end{tabular}

deviance of the model indicated good model fit (scaled

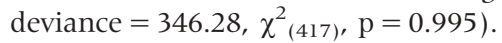

New or "incident" cases were excluded from the analysis of mean costs owing to their incomplete period of follow (less than six months). Mean duration of observation for these cases was 15.8 weeks. However, mean (95\% CI) costs for incident cases of Crohn's disease and ulcerative (or indeterminate) colitis were $£ 2662$ ( $£ 1006, £ 5866)$ and $£ 2111$ ( $£ 1488$, $£ 3078)$, respectively. These values are greater than the mean costs for prevalent cases, illustrating the relatively high costs of initial assessment and investigation of newly diagnosed cases of IBD.

\section{Costs of routine care versus active disease management}

Patients with quiescent IBD accounted for a total of 235 outpatient episodes (24\% of the total), costing $£ 17155$ (just $2.3 \%$ of total costs). Such patients may be suitable for alternative follow up arrangements (for example, on demand clinics or telephone consultations), with potential cost savings for secondary care clinics. In the case of ulcerative colitis, mean (95\% CI) six month cost of care for quiescent patients was $£ 359$ ( $£ 317, £ 469$ ) compared with $£ 765$ (£646, $£ 978$ ) for ambulatory patients suffering disease exacerbation and $£ 8861$ ( $£ 5725, £ 15$ 076) for those who were hospitalised during the study time frame. Corresponding values for Crohn's disease were $£ 275$ (£235, £319) for quiescent patients, $£ 578$ ( $£ 431, £ 701)$ for ambulatory patients suffering disease exacerbation, and $£ 5444$ (£3894, £9242) for those who were hospitalised. Hence disease flare up was associated with a 2-3-fold increase in six month costs for patients successfully managed as an outpatient but a more than 20 -fold increase in costs in the event of hospitalisation.

\section{Postal survey}

Questionnaires were returned by 233 patients (48\%). There was no difference between characteristics of responders and non-responders (see table 4). IBD sufferers reported significant interference with social activities, irrespective of the severity of the disease or disease type. Median (interquartile range (IQR)) lost days from "household and recreational activities" in six months was $17(8,55)$ for colitis and $20(9$, 60 ) days for Crohn's disease. Of this group of patients, $39 \%$ were in current employment. Thirty two per cent of employed ulcerative (or indeterminate) colitis sufferers reported loss of "employment days", with median (IQR) loss of earning over six months being £239 (£119, £597). Fifty per cent of employed patients with Crohn's disease had some loss of 
"employment days", with median (IQR) loss of earning over six months being $£ 299$ (£119, £597).

Mean (range) for out of pocket expenses (travel expenses, prescription charges, over the counter medications, extra clothing, and others) per six months was $£ 40(£ 0-£ 520)$ and $£ 66(£ 0-£ 750)$ for ulcerative colitis and Crohn's disease, respectively. The reported mean (range) of visits to the general practitioner was $1.08(0-8)$ and $1.33(0-10)$ per six months for ulcerative colitis and Crohn's disease, respectively. At a unit cost of approximately $£ 20$ per consultation, this amounts to an average cost of less than $£ 30$ per patient per six months for primary care visits.

\section{DISCUSSION}

The present study provides a cross sectional view of resource use among IBD patients attending a single centre. We believe this is the most comprehensive available record of the costs associated with IBD for the UK National Health Service. Although there are obvious limitations to the external validity of a single centre study, the characteristics of our patient cohort are very similar to those described for other parts of the UK in terms of demographics, crude incidence and prevalence rates, disease severity and extent, and hospitalisation rates. ${ }^{13}{ }^{20-27}$ Clearly, practice patterns may vary between different hospitals with respect to the use of specific pharmaceuticals or surgical techniques, and some larger tertiary centres will attract a patient population that is skewed towards more severely afflicted individuals.

There are significant obstacles to studying the costs of specific diseases in the UK. Diagnostic information is not routinely coded for most ambulatory care episodes and there is no requirement for recording of patient specific resource use under the current funding system. Hence our knowledge of the stream of resources consumed by individual patients with defined disease states is limited.

Costs of illness studies are inherently descriptive and do not seek to provide direct information about the relative cost effectiveness of different treatments or to test specific hypotheses. Nevertheless, by identifying and quantifying the relative contribution of different items and services to the overall economic burden of disease, such studies may provide important insights into the potential cost impact of new treatments or models of service delivery. Accurate primary data are also an essential requirement for theoretical modelling exercises if such techniques are to provide a credible alternative to formal trials that incorporate economic end points.

As with costs of illness studies from other countries, we used the prevalence approach to establish direct disease costs based on a retrospective analysis of routinely collected data. We aimed to identify all patients with IBD who accessed any form of relevant service at our centre during a fixed time interval. We wished to avoid the limitations imposed by a prospective patient capture design, which in practice results in the study of a convenient sample of patients such as those attending a particular clinic. Our cross referencing of multiple data sources allowed us to capture patients accessing care for a wide variety of purposes, including medical and surgical clinics, diagnostic procedures, and inpatient episodes. This included the full spectrum of disease, ranging from patients requiring hospitalisation and surgery through to nonconsulting patients whose only contact with the hospital might have been a routinely scheduled surveillance colonoscopy.

Reference to the existing literature indicates that our profile of UK resource use is very similar to that reported for other healthcare systems. The proportion of total direct healthcare costs attributed to hospitalisation (including surgery) has been estimated at $58 \%$ in Sweden ${ }^{4}$ and between
$55.8 \%^{7}$ and $57 \%^{8}$ in the USA compared with $49 \%$ in the present study. In agreement with these reports, only about $14 \%$ of our patient cohort were hospitalised during the study period. Estimates of the contribution of drugs to the total healthcare bill for IBD vary more widely, with a value of $24 \%$ reported for Sweden ${ }^{4}$ (similar to our own data) but values of just $3.5 \%{ }^{8}$ to $4.6 \%^{7}$ in North America. This probably reflects the proportionately higher costs of hospital care and physician fees compared with drugs in the USA compared with Europe. The potential impact of novel immunomodulating drugs (for example, anti-tumour necrosis factor therapy) on the profile of costs for IBD remains to be determined. No patient in our cohort received infliximab and our data therefore provide vital baseline information about the costs associated with established therapies for IBD.

A novel aspect of the present investigation is our examination of the clinical determinants of costs of care for IBD. A positive correlation between disease severity and costs of illness was to be expected, particularly as we graded disease severity by the intensity of medical treatment. However, the present study is the first to illustrate and quantify this relationship. A further novel aspect of the present analysis is our examination of variation in costs with disease extent. For both the main forms of IBD, average costs correlated positively with increased disease extent. However, this relationship was no longer apparent when disease severity was taken into account, resulting from the fact that disease extent was positively correlated with disease severity.

We have calculated the incremental cost of disease relapse compared with routine care of inactive disease at our institution, indicating that disease flares lead to a 2-3-fold increase in healthcare costs for those patients who were managed in an ambulatory setting. Hospitalisation led to a more than 20-fold increase in costs compared with quiescent disease. This reinforces the view that novel therapies capable of maintaining remission or reducing the need for inpatient care may prove cost effective despite their high acquisition costs when compared with other drug therapies. In addition to the obvious clinical benefits of minimising avoidable disease relapse, active measures aimed at maximising patient compliance with maintenance therapies may prove cost effective (for example, via patient education or more active specialist nurse supervision).

We also examined indirect costs associated with IBD by means of a cross sectional questionnaire survey. Between one third and one half of employed IBD patients reported some loss of work days during a six month period, similar to reports from Europe ${ }^{28} 29$ and North America. ${ }^{30} 31$ In addition, we estimated loss of time from household and recreational activities, an impact of IBD that has not been previously quantified. Our patients reported an average of 17-20 days of disrupted social activities per six months as a result of their illness. We also examined the level of non-reimbursable out of pocket expenses incurred by IBD patients. In the UK, patients pay fixed prescription charges rather than the full costs of medication such that the values for out of pocket expenses may appear low in comparison with those patients in other healthcare systems.

Based on our survey, the average frequency of consultations in general practice per six months was just 1.1 visits per patient. This is similar to values quoted in previous UK studies. ${ }^{132025}$ This is half the average rate of outpatient consultations in secondary (hospital) care, indicating that these diseases are managed largely in the secondary care setting in the UK. Inclusion of primary care visit costs to our costs of illness estimates would have added approximately $£ 30$ per patient per six months to average costs of care.

In conclusion, the present study provides a uniquely detailed description of costs of illness for IBD in the setting 
of the UK healthcare system. We confirm the major contribution of hospitalisation costs to the overall economic burden of these diseases and have quantified the economic impact of disease relapse. These cost data will assist future economic research into the cost effectiveness of rival therapies and management strategies for ulcerative colitis and Crohn's disease.

\section{ACKNOWLEDGEMENTS}

We would like to thank all the clinicians whose patients were included in the study, and in particular our gastroenterology colleagues (Dr RJ Walker, Professor N Krasner, Dr R Sturgess, Dr P ÒToole, Mr C Mackie, and Mr Dhorajewala).

Some of the administrative costs of the study were funded by donations from Procter and Gamble Ltd, UK, and Shire Pharmaceuticals, UK. KB and $\mathrm{AB}$ have received sponsorship to attend scientific meetings from these companies.

\section{Authors' affiliations}

A Bassi, Aintree Centre for Gastroenterology, University Hospital Aintree, Liverpool, UK

S Dodd, P Williamson, Centre for Medical Statistics and Health Evaluation, School of Health Sciences, University of Liverpool, Liverpool, UK

K Bodger, University Department of Medicine, Clinical Sciences Centre, University Hospital Aintree, Liverpool, UK

\section{REFERENCES}

1 Bodger K. Cost of illness of Crohn's disease. Pharmacoeconomics 2002; 20:639-52.

2 Ward FM, Bodger K, Daly MJ, et al. Clinical economics review: medical management of inflammatory bowel disease. Aliment Pharmacol Ther $1999 ; 13: 15-25$.

3 Silverstein MD, Loftus EV, Sandborn WJ, et al. Clinical course and costs of care for Crohn's disease: Markov model analysis of a population-based cohort. Gastroenterology 1999;1 17:49-57.

4 Blomqvist P, Ekbom A. Inflammatory bowel diseases: health care and costs in Sweden in 1994. Scand J Gastroenterol 1997;32:1134-9.

5 Pinchbeck BR, Kirdeikis J, Thomson AB. Economic impact of inflammatory bowel disease in Alberta. Can J Gastroenterol 1988;2:53-6.

6 Hay AR, Hay JW. Inflammatory bowel disease: medical cost algorithms. J Clin Gastroenterol 1992;14:318-27.

7 Hay JW, Hay AR. Inflammatory bowel disease: costs-of-illness. J Clin Gastroenterol 1992;14:309-17.

8 Feagan BG, Vreeland MG, Larson LR, et al. Annual cost of care for Crohn's disease: a payor perspective. Am J Gastroenterol 2000;95:1955-60.

9 Bernstein CN, Papineau N, Zajaczkowski J, et al. Direct hospital costs for patients with inflammatory bowel disease in a Canadian tertiary care university hospital. Am J Gastroenterol 2000;95:677-83.
10 Cohen RD, Larson LR, Roth JM, et al. The cost of hospitalization in Crohn's disease. Am J Gastroenterol 2000;95:524-30.

11 Netten A, Dennett J, Knight J. Unit cost of health and social care. Canterbury: Personal Services Research Unit (PSSRU), University of Kent, 1999, 2003.

12 Office for National Statistics. The new earning survey. London: Stationery Office, 2000.

13 Robinson A, Thompson DG, Wilkin D, et al. Guided self-management and patient-directed follow-up of ulcerative colitis: a randomised trial. Lancet 2001;358:976-81.

14 Davison AD, Hinkley DV. Bootstrap methods and their application. Cambridge: Cambridge University Press, 1997.

15 Hardin J, Hilbe J. Generalised linear models and extensions. Texas: Stata Press, 2001.

16 Hosmer DW, Lemeshow S. Applied logistic regression, 2nd ed. New York: John Wiley \& Sons, 1995

17 Box GEP, Cox DR. An analysis of transformations. J R Stat Soc 1964; (series B): $211-43$.

18 Park R. Estimation with heteroscedastic error terms. Econometrica 1966;34:888.

19 Efron B. Regression and ANOVA with zero-one data: Measures of residual variation. J Am Stat Assoc 2003;73:113-21.

20 Rubin GP, Hungin APS, Kelly PJ, et al. Inflammatory bowel disease: epidemiology and management in an English general practice population. Aliment Pharmacol Ther 2000;14:1553-9.

21 Primatesta P, Goldacre MJ. Crohn's disease and ulcerative colitis in England and the Oxford record linkage study area: a profile of hospitalized morbidity. Int J Epidemiol 1995;24:922-8.

22 Thompson NP, Fleming DM, Charlton J, et al. Patients consulting with Crohn's disease in primary care in England and Wales. Eur J Gastroenterol Hepatol 1998; 10:1007-12

23 Logan RF. Inflammatory bowel disease incidence: up, down or unchanged? Gut 1998:42:309-11.

24 Kennedy A, Nelson E, Reeves D, et al. A randomised controlled trial to assess the impact of a package comprising a patient-orientated, evidence-based selfhelp guidebook and patient-centred consultations on disease management and satisfaction in inflammatory bowel disease. Health Technol Assess 2003;7:iiil-113.

25 Williams JG, Cheung WY, Russell IT, et al. Open access follow up for inflammatory bowel disease: pragmatic randomised trial and cost effectiveness study. BMJ 2000;320:544-48

26 Stone MA, Mayberry JF, Baker R. Prevalence and management of inflammatory bowel disease: a cross-sectional study from central England. Eur J Gastroenterol Hepatol 2003;15:1275-80.

27 Nightingale AJ, Middleton W, Middleton SJ, etal. Evaluation of the effectiveness of a specialist nurse in the management of inflammatory bowe disease (IBD). Eur J Gastroenterol Hepatol 2000;12:967-73.

28 Rosch M, Leidl R, Tirpitz C, et al. (Cost measurement based on a cost diary in patients with inflammatory bowel disease). Z Gastroenterol 2002;40:217-28.

29 Boonen A, Dagnelie PC, Feleus A, et al. The impact of inflammatory bowel disease on labor force participation: results of a population sampled casecontrol study. Inflamm Bowel Dis 2002;8:382-9.

30 Longobardi T, Jacobs P, Wu L, et al. Work losses related to inflammatory bowel disease in Canada: results from a National Population Health Survey. Am J Gastroenterol 2003:98:844-849.

31 Longobardi T, Jacobs P, Bernstein CN. Work losses related to inflammatory bowel disease in the United States: results from the National Health Interview Survey. Am J Gastroenterol 2003;98:1064-72. 